\title{
A SAÚDE BUCAL EM PACIENTES DE UTI
}

\author{
Tarsila Spinola Pinheiro ${ }^{\alpha}$ \\ Tatiana Frederico de Almeida ${ }^{b}$
}

\begin{abstract}
Resumo
Introdução: As unidades de terapia intensiva (UTI) são voltadas ao cuidado do paciente em estado crítico em todos os níveis assistenciais. O cuidado com a saúde bucal tem se mostrado eficiente no controle de patógenos nesses pacientes e os protocolos de cuidados sistematizados são importantes para que seja provado o que é efetivo. Objetivos: Os objetivos desse estudo foram realizar uma revisão de literatura narrativa sobre Odontologia Hospitalar em UTI e uma revisão sistemática a respeito das publicações brasileiras sobre o tema. Metodologia: Foram selecionados estudos acerca da Odontologia Hospitalar em UTI publicados entre 2006 e 2013 no Brasil. As bases de dados empregadas para o rastreamento dos artigos foram LiLacs, BBO e SciELO, utilizando-se na busca a combinação das palavras "Odontologia" "Odontologia Hospitalar" e "UTI". Resultados: No rastreamento foram encontrados 12 artigos. Dos estudos selecionados, 04 foram revisões de literatura (33\%), 02 foram relatos de caso (17\%) e 06 foram estudos de corte transversal (50\%). Os estudos objetivaram sobretudo evidenciar a importância da presença do cirurgião dentista nas UTIs. Conclusões: As afecções bucais são importantes fontes de agravamento sistêmico e devem ser prevenidas e solucionadas por um cirurgião dentista capacitado a trabalhar em ambiente hospitalar. Os protocolos de conduta, quando utilizados em larga escala, trazem benefícios tanto ao paciente quanto ao hospital, reduzindo custos que agravamentos poderiam trazer.
\end{abstract}

Palavras-Chave: Saúde bucal; Unidade Hospitalar de Odontologia; Unidades de Terapia Intensiva.

\section{ORAL HEALTH IN ICU PATIENTS}

\begin{abstract}
Introduction: The intensive care unit are geared to take care of the patients in critical conditions and this requires attention in every level of assistance. The oral health care has shown efficiency in pathogens control at these patients and systematic protocols are important to prove how effective it is. Objective: The objectives of this study were to review the literature on narrative Dental Hospital ICU and a systematic review about Brazilian publications on the topic. Methods: We selected studies on
\end{abstract}

a. Graduada em Odontologia pela Escola Bahiana de Medicina e Saúde Pública. tarsilaspinolap@gmail.com

b. Professora do curso de Odontologia da Escola Bahiana de Medicina e Saúde Pública. tatifrederico@yahoo.com.br 
Dental Hospital ICU published between 2006 and 2013 in Brazil. The databases used for tracking items were LILACS, BBO and SCIELO, using the search combining the words "Dentistry", "Hospital Dentistry" and "ICU". Results: In the tracking were found 12 articles. On these selected studies, 04 were literature reviews (33\%), 02 were case reports (17\%) and 06 were cross-sectional studies (50\%). Studies had the intention of show the importance of the presence of the dentist in ICUs. Conclusions: The oral diseases are important sources of worsening systemic and must be prevented and solved by a dentist trained to work in a hospital setting. The protocols of conduct, when used on a large scale, brings benefits both the patient and the hospital, reducing cost escalations that would causes.

Keywords: Oral health; Dental Service Hospital; Intensive Care Units.

\section{INTRODUÇÃ̃O}

As unidades de terapia intensiva (UTIs) são voltadas às necessidades de atendimento do paciente cujo estado exige uma assistência e observação contínua dos profissionais de saúde. Equipes interdisciplinares e multidisciplinares fazem parte do dia a dia destas unidades. ${ }^{(1)}$

O cuidado para com o paciente internado deve ser avaliado de forma integral, envolvendo as áreas multidisciplinares da saúde. Deve ser prezado também o bem-estar geral de um paciente em cuidados intensivos. Uma sensação de conforto adequada seria fundamental para melhorar a sobrevida desses pacientes, porém, é de extrema dificuldade promover a qualidade de vida em um ambiente onde a preocupação mais eminente é a luta contra a morte. ${ }^{(2)}$

O paciente de UTI encontra-se em meio a uma situação que abrange dificuldades familiares e psicossociais que podem agravar o quadro clínico, assim, cuidados de caráter multidisciplinar devem ser abrangidos. (1)

A Odontologia Hospitalar é uma especialidade relativamente nova e que vai muito além da cirurgia bucomaxilofacial. São preconizados ao paciente em tratamento intensivo cuidados orais a fim de prevenir novas infecções em um ambiente tão crítico. Uma adequada avaliação odontológica pode determinar a necessidade e o tempo apropriados paraintervir em situações de riscos futuros de contaminação, e a adequação bucal pode transformar o desfecho clínico, reduzindo fatores que possam influenciar negativamente o tratamento sistêmico. (3)

A falta de adequada higiene bucal é propícia às condições de crescimento bacteriano. Uma maior quantidade e diferenciação do biofilme dental podem promover interações entre bactérias nativas e patógenos respiratórios, contribuindo para o desenvolvimento de doenças como a pneumonia.(4)

Os objetivos deste estudo foram realizar uma revisão de literatura narrativa sobre Odontologia Hospitalar em UTI e uma revisão sistemática a respeito das publicações brasileiras sobre o tema, a fim de sistematizar o conhecimento produzido sobre esta temática ainda tão recente na Odontologia, porém com tanta relevância para a qualidade de vida destes pacientes.

\section{REVISÃO DA LITERATURA}

\section{ODONTOLOGIA HOSPITALAR}

A Odontologia Hospitalar é uma especialidade odontológica que visa a realização de cuidados e 
procedimentos bucais em âmbito hospitalar. Os primeiros serviços odontológicos estabelecidos em um hospital aconteceram em 1901, no Hospital Geral da Filadélfia, e tiveram como função o cuidado dental dos pacientes e o treinamento de estudantes da área. ${ }^{(4)}$

Araújo, Vinagre e Sampaio em $2009^{(2)}$ relataram que indivíduos hospitalizados tendem a apresentar má higiene bucal e que essa ausência de atenção resulta no aumento e complexidade do biofilme dental.

A condição de higiene bucal deficiente desencadeia uma série de doenças bucais tais como a xerostomia, periodontite e gengivite potencializando focos de infecções que propiciam maior risco de complicações locais e sistêmicas. ${ }^{5,6)}$

De acordo com o Código de Ética Odontológico, ${ }^{(6)}$ compete ao cirurgião dentista especializado em Odontologia Hospitalar, internar e assistir pacientes em hospitais públicos e privados, com ou sem caráter filantrópico, respeitando as normas técnico-administrativas das instituições.

Segundo Camargo em 2005, ${ }^{(3)}$ o profissional especializado em Odontologia Hospitalar pode trabaIhar como consultor da saúde bucal e/ou prestador de serviços, seja em nível ambulatorial ou regime de internação, sempre com o objetivo de colaborar, oferecer e agregar forças ao que caracteriza a nova identidade hospitalar.

\section{RELAÇÃO ENTRE DOENÇAS BUCAIS E SISTÊMICAS}

As primeiras suspeitas de relações entre doenças bucais e sistêmicas nos artigos científicos datam 110 a.C.(7) Desde então, muitos pesquisadores se empenham em obter resultados que comprovem essa relação, indicando que problemas bucais como a periodontite atuam como foco de disseminação de patógenos com efeito sistêmico. ${ }^{(8,9)}$

A cavidade bucal representa o maior meio de comunicação do meio ambiente com o organismo, tendo cerca de 500 diferentes tipos de microrganismos colonizando a área. Com o imunocomprometimento do paciente, muitos destes microrganismos apresentam seu potencial patogênico aumentado, levando a doenças periodontais severas e candidíase. ${ }^{(10)}$

Entre as doenças sistêmicas com fator de risco para doença periodontal estão a diabetes, que nestes pacientes se manifesta de forma mais severa devido a fatores metabólicos e a AIDS que, por conta da deficiência imunológica, traz consigo uma série de infecções por fungos, vírus e bactérias ao paciente. ${ }^{(10)}$

A doença periodontal e a má condição de saúde bucal são fatores de risco para a doença cardíaca. O aumento do número de bactérias no interior do epitélio juncional pode resultar na penetração das bactérias e seus subprodutos nos tecidos gengivais, causando um processo inflamatório e todas as consequências deste. Oliveira et al. relataram no estudo que a presença de bactérias periodontais expõe o hospedeiro a uma variedade de eventos nocivos os quais podem predispor a doenças cardiovasculares. ${ }^{(11)}$

Dentre outras doenças sistêmicas que podem seradquiridas a partir da microbiota bucal estão as respiratórias, como a pneumonia. Em sua classificação, pode-se diferenciar a pneumonia quanto ao meio de desenvolvimento: quando adquiridas na comunidade ou nosocomiais, que se dão após $48 \mathrm{~h}$ de internação hospitalar. A pneumonia nosocomial pode se desenvolver a partir da aspiração de patógenos presentes na microbiota bucal ou a partir da doença periodontal, pela difusão hematológica dos patógenos presentes na microbiota bucal. ${ }^{(12)}$

\section{UNIDADE DE TERAPIA INTENSIVA}

Nascimento e Trentini (2004) pontuaram que a internação na UTI rompe bruscamente com o modo de viver do sujeito, incluindo suas relações e seus papéis e a sua identidade fica fortemente afetada. Devido a severidade do seu estado, o paciente não é considerado atuante de suas escolhas e geralmente não exercem a autônomia em coisas simples como a higiene pessoal, alimentação e excreção. ${ }^{(13)}$ 
Condições de deficiência da higiene bucal são muito comuns nesses pacientes, que frequentemente permanecem com a boca aberta devido à intubação traqueal. Isso promove a desidratação da mucosa e leva à diminuição do fluxo salivar, permitindo maior colonização de bactérias e levando a maior predisposição a doenças periodontais e outros possíveis focos de infecção. ${ }^{(5)}$

Em 2010 a Vigilância Sanitária publicou, no Diário Oficial da União, a Resolução da Diretoria Colegiada $\mathrm{N}^{\circ} 72010$, onde foram estipulados requisitos mínimos para o funcionamento de uma UTI. Neste ficam definidos os serviços que o paciente deve ter à beira do leito, sendo o VI tópico a assistência odontológica. Com isso, as UTIs devem se adequar no sentido que seu corpo assistêncial contenha pelo menos um profissional da área odontológica. ${ }^{(14)}$

Desta maneira, é imprescindível a atuação do profissional de Odontologia para o estabelecimento da prevenção e cuidados na saúde bucal do paciente. A interdisciplinaridade deve atuar em UTIs de maneira que a prevenção seja tão importante quanto a cura.

\section{ATUAÇÃO DO CIRURGIÃO DENTISTA EM UTI}

A condição clínica dos pacientes em estado crítico, principalmente aqueles em Ventilação Mecânica (VM), dificulta a higienização bucal, o que leva a uma proliferação bacteriana e formação de biofilme dentário. ${ }^{(15)}$

Os pacientes hospitalizados em UTIs muitas vezes estão totalmente debilitados e dependentes de cuidados, portanto impossibilitados de exercer sua higiene bucal, necessitando do suporte de profissionais de saúde para realizar tais procedimentos. A manutenção da saúde bucal visa o tratamento global do paciente, visto que a cavidade bucal é a primeira porta de entrada para microrganismos patogênicos respiratórios que causam infecções sistêmicas. ${ }^{(16)}$
A presença de biofilme interfere a partir de alterações bucais presentes no paciente, tais como cárie, doença periodontal, necrose pulpar, entre outros. Estas alterações repercutem tanto na terapêutica médica quanto no estado geral do paciente e devem ser controladas. ${ }^{(17)}$

Para que causas bucais não possam trazer consequências sistêmicas, é de extrema importância a presença de um cirurgião dentista no âmbito hospitalar para que possa ser feito o diagnóstico das alterações bucais e consequente auxílio da terapêutica médica; sejam em procedimentos de emergência como traumas ou abcessos, procedimentos que previnam o agravamento da doença sistêmica ou estabelecimento de uma infecção hospitalar e procedimentos curativos, como a adequação do meio bucal do paciente crítico. ${ }^{(17)}$

Além disso, cabe ao cirurgião dentista preparar uma equipe de Odontologia Hospitalar, incluindo nos procedimentos equipamentos, materiais e instrumentais adequados ao atendimento e um preparo profissional especializado. ${ }^{(9)}$

\section{PROTOCOLOS DE CUIDADOS COM A SAÚDE BUCAL EM PACIENTES DE UTI}

O investimento em implementação de protocolos de cuidado com a saúde bucal para diminuir riscos de doenças sistêmicas e infecções hospitalares é de grande valia para a saúde pública e privada. Medidas simples como escovar os dentes dos pacientes duas vezes ao dia e utilizar antissépticos bucais mostram redução da mortalidade e morbidade de pacientes em UTIs, entretanto, deve-se distinguir o paciente de acordo com o seu quadro clínico para que o devido protocolo seja utilizado. Pacientes conscientes ou entubados se diferenciam tanto no tipo de colonização microbiana da cavidade bucal quanto na terapêutica a ser utilizada. ${ }^{(18,19)}$

Pasetti et al. em 2013 definiram certos protocolos que objetivam o tratamento e controle das doenças bucais de forma rápida e adequada, a redução da morbi-mortalidade, a melhora na qualidade de vida e a diminuição no tempo de hospitalização. Dentre 
eles, o mais difundido é o Protocolo de Treinamento da Enfermagem para Higiene Oral. Neste, orienta-se o profissional de enfermagem a respeito da higienização mecânica através da escovação bucal, com a escova a $45^{\circ} \mathrm{em}$ direção ao colo dentário e o sulco gengival com ligeiras vibrações nos dentes posteriores e, além de higienização da mucosa com gaze úmida e escovação de língua. O controle químico se dá diferenciando pacientes com ou sem lesões na boca; nos primeiros deve-se administrar Digluconato de Clorexidina 0,12\% e nos ultimos deve-se administrar colutório bucal comum. A limpeza das próteses deve ser realizada com agua e sabão ou com dentifrício e escova dental média. ${ }^{(18,20,21)}$

Além da higiene bucal diária, existem protocolos específicos para intervenção e melhoria de casos como pacientes oncológicos, com candidíase, processo infeccioso oral, dentre outros. ${ }^{(21)}$

\section{METODOLOGIA}

Para realização da revisão sistemática de literatura de estudos nacionais sobre Odontologia Hospitalar em UTI, foram selecionados estudos acerca do tema publicados entre 2006 e 2013 no Brasil. As bases de dados empregadas para o rastreamento dos artigos foram LiLacs, BBO e SciELO, utilizando-se na busca a combinação das palavras "Odontologia", "Odontologia Hospitalar" e "UTI". Foram consideradas apenas publicações em português. Esta pesquisa bibliográfica foi finalizada em agosto de 2013.

Todos os resumos de artigos contendo as palavras-chave foram lidos e avaliados, e considerados relevantes aqueles que abrangiam o cirurgião dentista em âmbito hospitalar e atuando em pacientes das UTIs. O primeiro rastreamento identificou 15 , dos quais, apenas 12 se enquadravam nos critérios da pesquisa.

\section{RESULTADOS}

No rastreamento foram encontrados 15 artigos, dos quais 03 foram excluídos por não estarem de acordo com o requerido para esta revisão. Quanto à localização de realização dos artigos, a maioria deles aconteceu na região Sudeste, tendo como estado principal São Paulo (50\%). Entretanto, estudos também foram realizados nas regiões Norte, Nordeste e Sul. Em relação aos seus objetivos, grande maioria procurou demonstrar a necessidade e importância da atuação do cirurgião dentista na UTI (Quadro 1).

Quadro 1 - Resumo das publicações nacionais sobre Odontologia Hospitalar em UTI, no período de 2006 a 2013

\begin{tabular}{|c|c|c|c|c|}
\hline AUTOR & LOCAL/ANO & OBJetivos & METOdOLOGIA & Resultados \\
\hline Morais et al. & $\begin{array}{l}\text { SÃO PAULO, } \\
2006\end{array}$ & $\begin{array}{l}\text { Buscar dados na } \\
\text { literatura sobre a } \\
\text { participação da } \\
\text { condição bucal no } \\
\text { estabelecimento da } \\
\text { pneumonia nosocomial }\end{array}$ & $\begin{array}{l}\text { REVISÃO DE } \\
\text { LITERATURA }\end{array}$ & $\begin{array}{l}\text { Estudos ainda não estão } \\
\text { definidos, entretanto, } \\
\text { é imprescindível } \\
\text { a manutenção da } \\
\text { saúde bucal para a } \\
\text { prevenção de problemas } \\
\text { sistêmicos. }\end{array}$ \\
\hline Oliveira et al. & $\begin{array}{c}\text { RIO DE } \\
\text { JANEIRO, } 2007\end{array}$ & $\begin{array}{l}\text { Investigar a presença de } \\
\text { patógenos respiratórios } \\
\text { na cavidade oral de } \\
\text { pacientes em UTI }\end{array}$ & $\begin{array}{c}\text { ESTUDO } \\
\text { DE CORTE } \\
\text { TRANSVERSAL }\end{array}$ & $\begin{array}{l}\text { A presença de } \\
\text { patógenos no biofilme } \\
\text { bucal de pacientes } \\
\text { em UTI pode servir de } \\
\text { associação à pneumonia } \\
\text { nosocomia }\end{array}$ \\
\hline
\end{tabular}


Quadro 1 - Resumo das publicações nacionais sobre Odontologia Hospitalar em UTI, no período de 2006 a 2013

(continuação)

\begin{tabular}{|c|c|c|c|c|}
\hline AUTOR & LOCAL/ANO & OBJetivos & METODOLOGIA & Resultados \\
\hline Santos et al. & $\begin{array}{l}\text { SÃO PAULO, } \\
2008\end{array}$ & $\begin{array}{l}\text { Avaliar a eficiência de } \\
\text { solução enzimática } \\
\text { (Bioténe Mouthwash) } \\
\text { na higiene bucal de } \\
\text { pacientes dependentes } \\
\text { de cuidados em UTI }\end{array}$ & $\begin{array}{c}\text { ESTUDO } \\
\text { DE CORTE } \\
\text { TRANSVERSAL }\end{array}$ & $\begin{array}{l}\text { Não houve diferença } \\
\text { significativa entre os } \\
\text { grupos higienizados com } \\
\text { ou sem a solução }\end{array}$ \\
\hline Araújo et al. & BELÉM, 2009 & $\begin{array}{l}\text { Estabelecer um perfil } \\
\text { dos cuidados em saúde } \\
\text { bucal prestados a } \\
\text { pacientes de UTI por } \\
\text { equipes de enfermagem }\end{array}$ & $\begin{array}{c}\text { ESTUDO } \\
\text { DE CORTE } \\
\text { TRANSVERSAL }\end{array}$ & $\begin{array}{l}\text { Os cuidados de higiene } \\
\text { bucal são escassos } \\
\text { e inadequados, } \\
\text { sendo necessárias } \\
\text { modificações }\end{array}$ \\
\hline Araújo et al. & $\begin{array}{l}\text { MARINGÁ, } \\
2009\end{array}$ & $\begin{array}{l}\text { Demonstrar a atuação } \\
\text { de enfermeiros } \\
\text { em equipes } \\
\text { multidisciplinares } \\
\text { e questionar se a } \\
\text { presença do cirurgião } \\
\text { dentista é necessária } \\
\text { nessas equipes }\end{array}$ & $\begin{array}{c}\text { ESTUDO } \\
\text { DE CORTE } \\
\text { TRANSVERSAL }\end{array}$ & $\begin{array}{l}86 \% \text { dos entrevistados } \\
\text { acham necessária } \\
\text { a presença do } \\
C D \text { em equipes } \\
\text { multidisciplinares }\end{array}$ \\
\hline Rabelo et al. & $\begin{array}{l}\text { SÃO PAULO, } \\
2010\end{array}$ & $\begin{array}{l}\text { Relatar um caso de } \\
\text { paciente internada em } \\
\text { UTI que apresentou } \\
\text { lesões ulceradas em } \\
\text { mucosa jugal }\end{array}$ & $\begin{array}{c}\text { RELATO DE } \\
\text { CASO CLÍNICO }\end{array}$ & $\begin{array}{l}\text { A participação da } \\
\text { Odontologia na equipe } \\
\text { foi fundamental para a } \\
\text { indicação terapêutica }\end{array}$ \\
\hline Miranda et al. & $\begin{array}{l}\text { SÃO PAULO, } \\
2010\end{array}$ & $\begin{array}{l}\text { Abordar a intervenção } \\
\text { odontológica na UTI de } \\
\text { um hospital em Brasília }\end{array}$ & $\begin{array}{c}\text { RELATO DE } \\
\text { CASO CLIÍNICO }\end{array}$ & $\begin{array}{l}\text { A participação de um } \\
C D \text { com capacitação } \\
\text { nesse tipo de paciente } \\
\text { ajuda de fato numa } \\
\text { mudança dos casos } \\
\text { clínicos }\end{array}$ \\
\hline Barros et al. & $\begin{array}{c}\text { FORTALEZA, } \\
2011\end{array}$ & $\begin{array}{l}\text { Mostrar a importância } \\
\text { da presença do CD em } \\
\text { um hospital do SUS }\end{array}$ & $\begin{array}{c}\text { ESTUDO } \\
\text { DE CORTE } \\
\text { TRANSVERSAL }\end{array}$ & $\begin{array}{l}\text { A presença do CD } \\
\text { é necessária ao } \\
\text { diagnóstico e controle } \\
\text { das afecções bucais }\end{array}$ \\
\hline Slawski et al. & $\begin{array}{c}\text { PORTO } \\
\text { ALEGRE, } 2011\end{array}$ & $\begin{array}{c}\text { Avaliar a presença da } \\
\text { Odontologia nas UTIs } \\
\text { dos hospitais de Porto } \\
\text { Alegre }\end{array}$ & $\begin{array}{c}\text { ESTUDO } \\
\text { DE CORTE } \\
\text { TRANSVERSAL }\end{array}$ & $\begin{array}{l}\text { Revela que a atenção } \\
\text { na UTIs está voltada ao } \\
\text { cuidado de ferimento e } \\
\text { próteses }\end{array}$ \\
\hline Padovani et al. & $\begin{array}{c}\text { SÃO PAULO, } \\
2012\end{array}$ & $\begin{array}{c}\text { Organizar e administrar } \\
\text { as condutas } \\
\text { odontológicas que } \\
\text { tendem a prevenir } \\
\text { complicações sistêmicas } \\
\text { na UTI pediátrica }\end{array}$ & $\begin{array}{l}\text { REVISÃO DE } \\
\text { LITERATURA }\end{array}$ & $\begin{array}{l}\text { Diminuição das } \\
\text { infecções por } \\
\text { microrganismos da } \\
\text { cavidade bucal e } \\
\text { melhora da sobrevida } \\
\text { dos pacientes }\end{array}$ \\
\hline
\end{tabular}


Quadro 1 - Resumo das publicações nacionais sobre Odontologia Hospitalar em UTI, no período de 2006 a 2013

\begin{tabular}{|c|c|c|c|c|}
\hline AUTOR & LOCAL/ANO & OBjetivos & METODOLOGIA & REsultados \\
\hline Gomes et al. & $\begin{array}{l}\text { RIO DE } \\
\text { JANEIRO, } \\
2012\end{array}$ & $\begin{array}{l}\text { Buscar informações } \\
\text { sobre a Odontologia } \\
\text { Hospitalar no Brasil }\end{array}$ & $\begin{array}{l}\text { REVISÃO DE } \\
\text { LITERATURA }\end{array}$ & $\begin{array}{l}\text { OCD deve estar } \\
\text { presente e apto } \\
\text { para o atendimento } \\
\text { odontológico nos } \\
\text { hospitais }\end{array}$ \\
\hline Pasetti et al. & BAURU, 2012 & $\begin{array}{l}\text { Apresentar fatores } \\
\text { que influenciam o } \\
\text { estado do paciente } \\
\text { em UTI relacionados } \\
\text { ao tratamento } \\
\text { odontológico }\end{array}$ & $\begin{array}{l}\text { REVISÃO DE } \\
\text { LITERATURA }\end{array}$ & $\begin{array}{l}\text { A falta de conhecimento } \\
\text { tanto dos profissionais } \\
\text { de UTI em relação a } \\
\text { saúde bucal quanto do } \\
\text { CD em relação a UTI }\end{array}$ \\
\hline
\end{tabular}

Entre os estudos selecionados, o4 foram revisões de literatura (33\%) abrangendo a importância do cirurgião dentista na UTI, O2 foram relatos de caso $(17 \%)$ sobre a condição bucal e o tratamento de pacientes em UTI e 6 foram estudos de corte transversal (50\%) (Quadro 1).

No Quadro 1 pode-se observar os principais achados dos estudos analisados. Considerando os estudos de revisão da literatura, Morais et al. concluíram que não existe um conhecimento consolidado sobre a relação da condição bucal no estabelecimento da Pneumonia Nosocomial, já Gomes et al. concluíram que o cirurgião dentista deve estar presente e apto para o atendimento hospitalar. Padovani et al. sugeriram que cuidados bucais protocolados levam a diminuição das infecções por microrganismos da cavidade oral. . $9,16,22)$

Pasetti et al. também através de revisão da literatura e relato de experiência pessoal apresentaram estudo recente sobre fatores de relevância no tratamento odontológico de pacientes em UTI, traçando um perfil das patologias na UTI mais comumente adquiridas do Instituto de Neurologia de Curitiba-PR, como pneumonia nosocomial, lesões traumáticas e infecções da cavidade bucal. Os autores salientaram que há escassez de conhecimento dos profissionais da área de saúde a respeito de patologias e métodos de prevenção odontológicos, há deficiência na formação dos cirurgiões dentistas para atuação em pacientes sob terapia intensiva, além disso, se faz de suma importância a terapia preventiva (higiene bucal) como método de redução da incidência da pneumonia nosocomial. Para eles, uma equipe multidisciplinar atuando em prol do paciente internado em UTI propicia o correto diagnóstico e tratamento destes dos casos. ${ }^{(18)}$

Em seu relato de caso, Rabelo et al. concluíram que a atuação odontológica favoreceu a adequada indicação terapêutica do caso abordado. Miranda et al.em um outro relato de caso clínico de um hospital no Distrito Federal, observaram uma melhora significativa do quadro clínico do paciente após a interevenção do cirurgião dentista, além da diminuição da possibilidade de instalação das doenças oportunistas. ${ }^{(17,21)}$

Em relação aos estudos quantitativos de corte transversal, todos foram de caráter descritivo. Araújo et al. estabeleceram um perfil dos cuidados orais realizados pela equipe de enfermagem em UTIs e chegaram a conclusão que tais cuidados são escassos e inadequados, sendo necessárias modificações. Em outra pesquisa realizada por meio de entrevista com questionários Araújo et al. buscaram demonstrar o que acham a equipe de enfermagem a respeito da atuação de cirugiões dentistas em equipes multidisciplinares atuantes em 
UTIs e se a presença dos mesmos é de fato necessária. Como resultado deste inquérito, $86 \%$ dos entrevistados acreditavam ser necessária a presença de cirurgiões dentistas nas equipes multidisciplinares. ${ }^{(1,2)}$

Santos et al. ${ }^{(5)}$ em um estudo de corte transversal piloto avaliaram a eficiência da ação antimicrobiana da solução enzimática associada a higiene bucal em pacientes dependentes de cuidados internados em UTI e verificaram que o uso de solução enzimática (Bioténe Mouthwash - MS 2.2561.0003.001-6) se mostrou eficiente clinicamente na higiene bucal destes pacientes. Foi salientada a necessidade de serem desenvolvidas mais pesquisas sobre os cuidados bucais destes indivíduos.

Oliveira et al. ${ }^{(12)}$ em um outro estudo de corte transversal investigaram a presença de patógenos respiratórios na cavidade bucal de pacientes em UTI. Foram incluídos no estudo 30 pacientes com idades entre 18 e 82 anos internados na UTI geral do Hospital Municipal Raul Sertã em Nova Friburgo-RJ e diagnosticados com pneumonia nosocomial e a partir disto foram realizadas culturas e amostras microbiológicas da placa dental supragengival, da língua e do tubo orotraqueal. Os autores concluiram que a presença de patógenos respiratórios no biofilme bucal dos pacientes pode servir de reservatório para microrganismos relacionados com a pneumonia nosocomial.

\section{DISCUSSÃO}

Nesta revisão da literatura observa-se que a Odontologia em UTI está cada vez mais sendo reconhecida como fundamental à saúde do paciente internado. Na prática, a Odontologia Hospitalar tem sido concentrada na área de Cirurgia Bucomaxilofacial.(3)

As doenças bucais e sistêmicas estão diretamente interligadas. De acordo com Doro et al., ${ }^{(9)}$ a doença periodontal é fator de risco para diabetes e complicações cardíacas. De Souza et al. ${ }^{(10)}$ corroboram com a idéia de que o aumento de bactérias expõe o hospedeiro a uma variedade de eventos nocivos que podem predispor a doenças cardiovasculares.

Conforme os resultados analisados na revisão sistemática da literatura observou-se a predominância de estudos de corte transversal descritivos sobre a Odontologia Hospitalar em UTIs. Araújo et al.em ambos os seus estudos de 2009, demostraram a percepção de equipes de enfermagem a respeito da necessidade do cirurgião dentista no atendimento multidisciplinar ao paciente crítico, esclarecendo o quanto essa participação aumentaria a qualidade de vida dos pacientes. Padovani et al., (20) apesar de estarem lidando com uma UTI neonatal, obtiveram evidências clínicas similares, onde os cuidados bucais protocolados diminuíram as infeccões por microrganismos e melhoraram a sobrevida dos recém-nascidos. . $^{(1,2)}$

Santos et al. ${ }^{(5)}$ e Oliveira et al. ${ }^{(1)}$ abordaram também em estudos de corte transversal a relação entre os patógenos da cavidade oral e a pneumonia nosocomial. Para Oliveira et al. a colonização do biofilme bucal por patógenos respiratórios traz o agravamento da condição bucaldo paciente e deve ser controlada através de procedimentos específicos. Já Santos et al., sugeriram que o uso de solução bucal com sistema enzimático reduz o nível inflamatório das gengivas e diminui a quantidade da microbiota patogênica.

Barros et al. ${ }^{(21)}$ e Slawski et al. ${ }^{(22)}$ avaliaram a presença do cirurgião dentista em UTIs. Barros et al. demonstraram a importância da presença do cirurgião dentista neste local, enquanto Slawski et al. avaliaram a sua atuação. Ambos os estudos sustentam a necessidade do profissional da Odontologia na área em questão.

Morais et al. ${ }^{(8)}$ evidenciam que a participação da condição bucal no estabelecimento da pneumonia nosocomial não está totalmente definida. Outrora, Gomes et al. afirmam que a condição bucal dos pacientes internados em UTIs necessita do acompanhamento de um cirurgião dentista habilitado em Odontologia Hospitalar. Pasetti et al. confirmaram a necessidade do cirurgião dentista em âmbito hospitalar, já que os pacientes não possuem uma 
higienização bucal adequada e controle de placa eficiente. $(9,16,18)$

Nos relatos de caso analisados, tanto Rabelo et al., ${ }^{(16)}$ quanto Miranda et al. ${ }^{(25)}$ relataram que a participação da Odontologia foi de fundamental importância para a terapêutica e ajuda dos casos abordados.

Em suma, grande parte dos autores mencionados concorda que são necessários mais estudos a respeito da integração do cirurgião dentista no âmbito hospitalar e confirmam a relação entre a condição bucal e doenças sistêmicas. Diante disso, todos concordam que esta integração está diretamente ligada a melhor sobrevida do paciente e diminuição significativa do agravamento de casos.

Do ponto de vista da produção do conhecimento, os autores ressaltaram a necessidade de mais estudos e trabalhos nesta área. Dentre os limites dos estudos analisados nesta revisão sistemática, foi verificado um grande número de trabalhos descritivos, de corte transversal, com amostras pequenas. Metodologias analíticas mais sofisticadas devem ser adotadas em estudos longitudinais com amostras maiores de pacientes.

\section{CONCLUSÕES}

Ao término desta revisão de literatura podemos concluir que as afecções bucais são importantes fontes de agravamento sistêmico e que devem ser prevenidas e solucionadas por um cirurgião dentista capacitado a trabalhar em ambiente hospitalar. A temática ainda recente condiz com a pouca quantidade de artigos científicos nacionais encontrados na revisão sistemática da literatura sobre o tema pesquisado, o que leva a necessidade de abordar cada vez mais o assunto na Odontologia, inclusive durante a graduação. A multidisciplinaridade contida nas UTIs não se faz completa sem a presença do cirurgião dentista, já que grande maioria dos profissionais de saúde que fazem parte do corpo clínico de UTIs não dispõe do conhecimento necessário para o diagnóstico e tratamento de quais- quer condições anormais da cavidade bucal. Os estudos nacionais analisados concluiram que além de propor protocolos clínicos de conduta a esses pacientes, os cirurgiões-dentistas devem estar presentes e capacitados para atuar nos hospitais.

\section{REFERÊNCIAS}

1. Araújo RJG, Oliveira LCG, Hanna LMO, Corrêa AM, Carvalho LHV, Alvares NCF. Análise de percepções e ações de cuidados bucais realizados por equipes de enfermagem em unidades de terapia intensiva. Rev. bras. ter. Intensiva. 2009;21(1):38-44.

2. Araújo RJG, Vinagre NPL, Sampaio JMS. Avaliação sobre a participação de cirurgiõesdentistas em equipes de assistência ao paciente. Acta sci., Health sci. Maringá. 2009;31(2): 153-7.

3. Camargo EC. Odontologia hospitalar é mais do que cirurgia buco-maxilo-facial. Jornal do Site [publicação de internet] 2005 [acesso em 25 de ago 2012]. Disponível em: http://www. odontologiamt.com.br/procedimentos/index. aspicod $=2$

4. Willis PJ. The role of Dentistry in the hospital. J Am Dent Soc Anesthesiol. 1965;12(1): 4O-4.

5. Santos PSS, Mello WR, Wakim RCS, Paschoal MAG. Uso de solução bucal com sistema enzimático em pacientes totalmente dependentes de cuidados em Unidade de Terapia Intensiva. Rev. bras. ter. Intensiva. 2009;2O(2):154-9.

6. Conselho Federal de Odontologia. Código de Ética Odontológico. Brasília; 2O12. Cap. XI, Artigo 26.

7. Morais TMN, Silva A, Avi ANRL, Souza PHR, Knobel E, Camargo LFA. A importância da atuação odontológica em pacientes internados em unidades de terapia intensiva. Rev. bras. ter. intensiva. 2006;18(4):412-7.

8. Reilly PG, Glaffey NM. História da sepsia bucal como causa de doenças. In: Williams RC, Offenbacher S. Periodontologia 2000. São Paulo: Ed. Santos; 2005. p. 13-8. 
9. Doro GM, Fialho LM, Losekann M, Pfeiff DN. Projeto "Odontologia Hospitalar" Aperfeiçoamento do conhecimento técnico necessário em situações de risco a vida do paciente e levantamento das condições de saúde bucal de pacientes hospitalizados. Rev. ABENO, 2006;6(1):49-53.

10. De Souza ELB, Lopes JCA, Junior AAG, Silva KLM, Silva ARS, Silva EF et al. A doença periodontal como fator de risco para doenças cardiovasculares. Int. J. Dent. 2006; 1(2).

11. Oliveira LCBS, Carneiro PPM, Fischer RG, Tinoco EMB. A presença de patógenos respiratórios no biofilme bucal de pacientes com pneumonia nosocomial. Rev. bras. ter. intensiva. 2007;19(4):428-33.

12. Nascimento ERP, Trentini M. O cuidado da enfermagem na Unidade de Terapia Intensiva (UTI): Teoria humanistica de Paterson e Zderad. Rev. Lat Am Enfermagem. 2004;12(2):250-7.

13. Sannapieco FA. Relação entre doença periodontal e doenças respiratórias. In: Rose LE, Genco RJ, Mealy BL et al. Medicina Periodontal. São Paulo: Ed. Santos, 2002. p. 83-97.

14. Pace MA. Avaliação Clínica e microbiológica da cavidade bucal de pacientes críticos com entubação orotraqueal de um hospital de emergência [dissertação]. Ribeirão Preto: Universidade de São Paulo; 2007.Gomes SF, Esteves MCL. Atuação do cirurgião dentista em UTI: um novo paradigma. Rev. bras. odontol. 2012;69(1):67-70.

15. Rabelo GD, Queiroz Cl, Santos PSS. Atendimento odontológico ao paciente em unidade de terapia intensiva. Arq méd. hosp. Fac. Ciênc. Méd. Santa Casa São Paulo, 2010;55(2):67-70.

16. Kahn S, Garcia CH, Junior JG, Namen FM, Machado WAS, Silva Junior JA. Avaliação da existência de controle da infecção oral nos pacientes internados em hospitais do estado do Rio de Janeiro. Ciênc. saúde coletiva. 2008;13(6):1825-1831.

17. Pasetti LA, Carneiro Leão MT, Araki LT, Albuquerque AMN, Ramos TMB, Santos SF et al. Odontologia Hospitalar A Importância do Cirurgião Dentista na Unidade de Terapia Intensiva. Rev. Odontologia (ATO). 2013;13(4):211-226.

18. Departamento de Odontologia. Associação de Medicina Intensiva Brasileira. Porto Alegre; 2011 Ago 11.

19. Padovani MCRL, Souza SAB, Sant'Anna GR, Guaré RO. Protocolos de cuidados bucais na Unidade de Tratamento Intensivo (UTI) Neonatal. Rev. bras. pesq. Saúde. 2012;14(1):7180 .

2O. Barros CS, Pimentel GG, Bezerra MMM, Aguiar Filho NB, Santos ES. Atuação Odontológica na Unidade de Terapia Intensiva (UTI) do Hospital Geral de Fortaleza (HGF). Perionews. 2011;5(3):271-5.

21. Slawski EG, Moreira IB, Junger R, Rosing CK. A participação do cirurgião-dentista na equipe das Unidades de Terapia Intensiva (UTI). Perionews. 2012;6(1):39-44.24.

22. Assis C. Atendimento Odontológico nas UTIs. Rev. Bras. Odontol. 2012;69(1):72-5.

23. Godoi APT, Francesco AR, Duarte A, Kemp APT, Silva-Lovato $\mathrm{CH}$. Odontologia Hospitalar no Brasil. Uma visão Geral. Rev. Odontol. UNESP, 2009;38(2):105-9.

24. Miranda AF, Montenegro FLB. Ação odontológica preventiva em paciente idoso dependente na Unidade de Terapia Intensiva (UTI) - Relato de Caso. Rev. Paul. Odontol. 2010;32(1):34-8. 